\title{
SARS-CoV2 at the Alveolar Epithelial-Endothelial Interface - A Hypothesis for the Pathogenesis of COVID-19
}

\author{
Francia Carolina Acosta Saltos ${ }^{1}$ and Alejandro David Acosta Saltos ${ }^{1,2}$ \\ 1. Lansdell Research House, London, United Kingdom \\ 2. Chelsea and Westminster Hospital, London, United Kingdom
}

Correspondence to: Dr Alejandro David Acosta Saltos, MBBS PhD, Emergency Medicine Department, Chelsea and Westminster Hospital, London, United Kingdom, Email:

AlejandroDavid.AcostaSaltos@chelwest.nhs.uk

\section{Abstract}

Severe COVID-19 is associated with viraemia and multiple organ disease. Similar clinicopathological features have been previously seen in SARS and MERS. Clinically, the severity of SARS, MERS and COVID-19 has been associated with the presence of SARSCoV, MERS-CoV or SARS-CoV2 viraemia in affected patients. In vitro work has looked at the pattern of viral entry and release from polarised epithelial cells infected by coronaviruses. This work has demonstrated a correlation between the severity of a coronavirus infection and the ability of the virus to reach and infect the basal surface of host cells. It has been postulated that this ability helps the virus invade the bloodstream of the host, resulting in a systemic infection with multiple organ involvement. Here we propose that basal surface release and entry of COVID-19 into and out of cells at epithelial-endothelial interface plays a key pathogenic role in severe COVID-19 disease.

\section{Background}

The breach of the epithelial-endothelial barrier at the alveoli appears to be a critical step for the development of severe COVID-19 infection. It has been previously suggested that immune and endothelial dysfunction may play an important role in this process. It has been postulated that the breach of the epithelial-endothelial barrier begins with inflammation and the enrolment immune cells into the alveolar cavity. From there, it is hypothesised, that SARS-CoV2 infected immune cells invade the bloodstream through a leaky endothelium $(1,2)$. Thus, providing a pathway through which viraemia and systemic disease may occur in severe COVID-19. 
However, little has been said about the pattern of SARS-CoV2 entry and release from infected cells at alveolar epithelial-endothelial interface. Here, based on clinical, pathological, cellular and molecular evidence we suggest that in severe COVID-19 disease critical pathogenic events may occur at the alveolar supportive tissue where epithelium and endothelium meet.

\section{Viraemia and multiple organ involvement are associated with severe SARS,} MERS and COVID-19

Previously, the human coronaviruses SARS-CoV and MERS-CoV have caused the international SARS and MERS epidemics, respectively $(3,4)$. Clinically COVID-19 resembles SARS and MERS, with most cases of these infections showing a self-limiting respiratory infection and a minority of cases showing severe multi-organ disease (3-6). Like COVID-19, SARS and MERS are associated with viraemia (7-10).

Interestingly, viraemia in SARS, MERS and COVID-19 correlates with poorer clinical outcomes. The extent of SARS-CoV2 RNAaemia is closely correlated with level of the proinflammatory cytokine IL-6, a marker of COVID-19 disease, and poorer prognosis (11). Similarly, SARS-CoV RNAaemia was previously found to be associated with prolonged illness (12) and admission to an intensive care unit (9). Furthermore, MERS-CoV RNAaemia has been correlated with markedly increased risk of death (8). It is important to note, that MERS infection is associated with a greater degree of mortality and viraemia compared to COVID19. Thus, While SARS-CoV2 RNAaemia was found in 15\% of hospitalised COVID-19 patients (11), MERS-CoV2 RNAaemia was reported in $30 \%$ of cases (8).

Thus, evidence from SARS, MERS and Covid-19 clinical research suggests that viraemia may play an important pathogenic role in severe disease.

\section{Histology of the alveolar wall facilitates the infection of different cell types}

The alveolar wall consists of three elements: surface epithelium, supporting tissue and blood vessels. Surface epithethilium is made up of type I and II alveolar epithelial cells. The supporting tissue is formed by the basement membrane (fine reticular, collagenous and elastic fibres) and occasional fibroblasts. Blood vessels are mainly capillaries made up of endothelial cells (13).

The alveolar walls are defended locally by alveolar macrophages that reside in the alveolar cavity. Blood born dendritic cells also play a role in detecting antigens in the alveolar cavity but to do this they extend processes from the supporting tissue layer where they can temporarily reside (14). In addition, neutrophils, macrophages and lymphocytes can be recruited to the alveolar wall through the vasculature during infection (2). 
The supporting tissue in the alveolar walls forms an attenuated layer beneath the epithelium and surrounding blood vessels. In most of the alveolar wall, the basement membrane which supports the capillary endothelium is directly applied to the basement membrane supporting the alveolar epithelium (13). At these points, only 100nm separates the basal surface of epithelial cells and endothelial cells in the alveoli (15).

Post-mortem studies of SARS cases looking at the cellular distribution of SARS-CoV infection have shown that the main target of the virus is alveolar epithelial cells $(16,17)$. Type II alveolar epithelial cells appear to be preferentially affected compared to type I (16). However, other cell types, including alveolar macrophages, lymphocytes and fibroblast, also demonstrate evidence of SARS-CoV replication within them. Importantly, endothelial cells are positive for intracellular SARS-CoV RNA demonstrating that, despite not been in direct contact with the air rich alveolar cavity, they have been infected by the virus (7). Interestingly, although there is post-mortem evidence that SARSC-CoV2 is primarily infects in alveolar epithelial cells (18), a recent study has also demonstrated evidence of SARS-CoV2 RNA in the endothelial cells in the capillary beds of multiple organs of COVID-19 cases (19).

Therefore, the histology of the alveolar wall facilitates the infection of different cell types.

\section{Pattern of SARS-CoV, MERS-CoV and SARS-CoV2 receptor expression is} consistent with multiple organ infection

The human coronaviruses (CoVs), including SARS-CoV, SARS-CoV2 and MERS-CoV, have a common basic structure. CoV particles are made up of their positive sense RNA genome and the structural proteins coded by it. Structural proteins include spike protein (S), envelope protein (E), membrane protein (M) and nucleocapsid protein $(\mathrm{N})$. The S-protein is the dominant surface protein of $\mathrm{CoV}$ and it mediates virus attachment to the host cell membrane during infection (20).

The S-proteins in CoVs bind to specific cell surface receptors in target cells to initiate infection. SARS-CoV and SARS-CoV2 bind the angiotensin converting enzyme (ACE2) $(21,22)$, which is found expressed in type I and II alveolar epithelial cells, as well as endothelial cells in the alveoli. Interestingly, ACE2 is also expressed in multiple organs, including intestine, liver, kidney and brain (23-25). MERS-CoV binds dipeptidyl peptidase 4 (DDP4) to cause infection (26). Like ACE2, DDP4 is found in alveolar pneumocytes and endothelium (26) as well as in multiple organs (27).

The pattern of ACE2 and DPP4 expression is consistent with the potential for COVID-19, SARS and MERS to produce multiple organ pathology. However, systemic involvement is restricted to a minority of cases infected by the viruses. Furthermore, MERS demonstrates a 
greater degree of systemic involvement compared to SARS and COVID-19 (28). Thus, multiple organ pathology may not be fully explained simply by the pattern of expression of ACE2 and DPP4 at the tissue level.

\section{The pattern of viral entry and release into and from polarised epithelial cells correlates with the severity of coronavirus infection}

In vivo, alveolar epithelial and endothelial cells are polarised cells. They have their basal cell surfaces attached to a basement membrane, while their apical surfaces interact with either the alveolar cavity or bloodstream, respectively (15).

In vitro work has been carried out for more than 25 years looking at the pattern of coronavirus entry and release into and from polarised epithelial cells (29). This work uses a cell culture method that aims to model the polarised nature of epithelial cells in vivo (30). Studies using this method suggest that there may be a correlation between the severity of a coronavirus infection and the ability of the virus to reach and infect the basal surface of host cells (31). It has been postulated that this ability helps the virus invade the bloodstream of the host, resulting in a systemic infection with multiple organ involvement (29). Evidence of this hypothesis comes from studies of both animal and human coronaviruses.

The entry and release of some animal CoVs occurs preferentially at the apical surface of polarised epithelial cells. CoVs such as transmissible gastroenteritis coronavirus (TGEV), bovine CoV (BCoV) and porcine epidemic diarrhoea virus (PEDV), which cause localised gastrointestinal infections in animals, predominantly infect polarised epithelial cells at their apical domain (30,32-34). After replication, these CoVs, which cause illness without systemic spread, are also mostly released from the apical domain of infected polarised cells. Thus, CoVs that produce localised infections may be associated with an apical pattern of entry to and release from target cells.

Although some animal CoVs demonstrate predominantly apical entry and release, there are others that show a preference for basal release. CoVs that cause severe systemic illness, such mouse hepatitis virus (MHV), feline coronavirus (FCoV) and canine coronavirus (CCoV), mainly infect polarised epithelial cells at their apical domain $(31,35,36)$. After replication, these CoVs, which invade the blood to cause disease in multiple organs, are mostly released from the basal domain. Therefore, basal release may be associated with systemic infections.

Human CoVs are preferentially released from the apical surface of infected cells. CoVs that cause mild localised respiratory illnesses in humans, CoV-229E and CoV-HKU1, as well as those that can cause disease in multiple organs, SARS-CoV and MERS-CoV, are mostly released from the apical domain of polarised human epithelial cells (37-42). Consequently, a 
preference for apical viral release appears to be a common feature in human CoVs, irrespective of whether they cause local or systemic disease.

Although human CoVs involved in systemic disease mostly exit from the apical domain, basal release is also evident. Tao et al. (2013) studying MERS- and SARS-CoV demonstrated that about a third of all virus left polarised epithelial cells via the basal surface. Although clinically, MERs causes more severe disease progression than SARS-CoV, there was no difference in extent of release at the basal domain of epithelial cells between both viruses (40). Thus, the potential for systemic invasion of a human CoV may not depend solely on basal release of viral particles in humans.

SARS- and MERS-CoV differ in their ability to enter the basal surface of epithelial cells. Tao et al. (2013) demonstrated that while SARS-CoV could only infect polarised epithelial cells via the apical surface, MERS-CoV could infect both the apical and basal domains of these cells. In fact, MERS-CoV infection through the basal surface produced a higher viral titre than apical infection (40). Therefore, difference in systemic invasion may depend on the ability of CoV to infect polarised by entering their basal surface.

The pattern of entry and release of CoVs from epithelial cells and their association with disease is complex. However, there is evidence that apical entry and release of CoVs from infected polarised cells may be associated with localised infections only. In contrast, basal entry and release of CoVs from infected polarised cells may be associated with systemic disease and multiple organ involvement.

\section{Conclusion}

If the pattern of SARS-CoV2 entry and release into and from infected epithelial cells in vivo resembles that seen in polarised epithelial cell culture experiments with other coronavirus, then a pathogenic mechanism for COVID-19 may be derived.

Here we propose that epithelial cells infected in COVID-19, release SARS-CoV2 from both their apical and basal surface. Apical viral release propagates infection primarily to the alveolar cavities and from there to the outside. In contrast, basal release spreads infection internally into the basement membrane and associated alveolar supportive tissue. At the alveolar supportive tissue, SARS-CoV2 might enter fibroblasts, dendritic cells, neutrophils and T-cells. Importantly, endothelial cells might also directly become infected by SARS-CoV2 released from the basal surface of epithelial cells. In this way, viraemia may occur as a direct consequence of SARS-CoV2 endothelial infection and may precede or may occur simultaneously as the infection of blood-born immune cells in COVID-19. 


\section{Future Research}

According to the in vitro evidence described above the extent that SARS-CoV2 spreads internally into the basement membrane and alveolar supportive tissue may depend on how much virus gets released from the basal surface of infected epithelial cells. However, it may also depend on the susceptibility of polarised epithelial or endothelial cells to be infected through the basal surface. Research looking at SARS-CoV2 infection of polarised endothelial cells in vitro might help to elucidate the mechanism proposed here further (43).

Understanding the pattern of expression of SARS-CoV2 receptors in the basal surface of epithelial and endothelial cells may shed more light on the processes driving SARS-CoV2 endothelial infection and viraemia. In this vein, it is important to know that receptors other than ACE2, such as GRP78 (44), DPP4 (45) and CD147 (46), have been suggested to be targets of SARS-CoV2. Research on the pattern of expression of these receptors and their role in COVID-19 may be fruitful (43).

In addition, studying the intracellular mechanism regulating the basal surface release of SARS-CoV2 from polarised epithelial cells might clarify what drives severe COVID-19 pathology. In this vein, recent work has suggested that the non-structural protein nsp7 interacts with Rab proteins, a family of intracellular transport regulators, in SARS-CoV2 infected cells (47). Targeting therapies to nsp7 may be a novel avenue of treatment.

Finally, focusing research on the interface between epithelial and endothelial may draw our attention to therapies that target the basement membrane and supportive tissue in the alveoli such as those used for pulmonary fibrosis (48). Targeting pharmacological therapies to the critical areas of pathology may result in more effective treatment for COVID-19.

\section{Contributors}

ADAS carried out the literature search and drafted the paper. ADAS and FCAS reviewed the evidence and formed the hypothesis. FCAS revised the manuscript. ADAS and FCAS have reviewed the paper prior to submission. This manuscript has not been currently accepted or published elsewhere.

\section{Declaration of interests}

We declare no competing interests.

\section{Acknowledgments}

We are grateful to those publishers who provided unrestricted and timely access to the literature reviewed in this paper. We dedicate this work to the front-line workers who put their life at risk to help others in such unprecedented times. 


\section{References}

1. Li H, Liu L, Zhang D, Xu J, Dai H, Tang N, et al. SARS-CoV-2 and viral sepsis: observations and hypotheses. The Lancet. 2020 May 9;395(10235):1517-20.

2. Teuwen L-A, Geldhof V, Pasut A, Carmeliet P. COVID-19: the vasculature unleashed. Nature Reviews Immunology. 2020 May 21;1-3.

3. Arabi YM, Balkhy HH, Hayden FG, Bouchama A, Luke T, Baillie JK, et al. Middle East Respiratory Syndrome. New England Journal of Medicine. 2017 Feb 9;376(6):584-94.

4. Peiris JSM, Yuen KY, Osterhaus ADME, Stöhr K. The Severe Acute Respiratory Syndrome. New England Journal of Medicine. 2003 Dec 18;349(25):2431-41.

5. Arabi YM, A. Arifi A, H. Balkhy H, Najm H, S. Aldawood A, Ghabashi A, et al. Clinical Course and Outcomes of Critically III Patients With Middle East Respiratory Syndrome Coronavirus Infection. Annals of Internal Medicine [Internet]. 2020 Mar 8 [cited 2020 May 19]; Available from: https://www.acpjournals.org/doi/abs/10.7326/M13-2486

6. Yin C, Wang C, Tang Z, Wen Y, Zhang S, Wang B. Clinical analysis of multiple organ dysfunction syndrome in patients suffering from SARS. Zhongguo Wei Zhong Bing Ji Jiu Yi Xue. 2004 Nov;16(11):646-50.

7. Gu J, Korteweg C. Pathology and Pathogenesis of Severe Acute Respiratory Syndrome. Am J Pathol. 2007 Apr;170(4):1136-47.

8. Kim SY, Park SJ, Cho SY, Cha R, Jee H-G, Kim G, et al. Viral RNA in Blood as Indicator of Severe Outcome in Middle East Respiratory Syndrome Coronavirus Infection. Emerg Infect Dis. 2016 Oct;22(10):1813-6.

9. Ng EKO, Hui DS, Chan KCA, Hung ECW, Chiu RWK, Lee N, et al. Quantitative Analysis and Prognostic Implication of SARS Coronavirus RNA in the Plasma and Serum of Patients with Severe Acute Respiratory Syndrome. Clin Chem. 2003 Dec 1;49(12):197680.

10. Wang W-K, Fang C-T, Chen H-L, Yang C-F, Chen Y-C, Chen M-L, et al. Detection of Severe Acute Respiratory Syndrome Coronavirus RNA in Plasma during the Course of Infection. Journal of Clinical Microbiology. 2005 Feb 1;43(2):962-5.

11. Chen X, Zhao B, Qu Y, Chen Y, Xiong J, Feng Y, et al. Detectable serum SARS-CoV-2 viral load (RNAaemia) is closely correlated with drastically elevated interleukin 6 (IL-6) level in critically ill COVID-19 patients. Clin Infect Dis [Internet]. 2020 Apr 17 [cited 2020 May 18]; Available from: https://academic.oup.com/cid/advancearticle/doi/10.1093/cid/ciaa449/5821311

12. Chen $\mathrm{W}, \mathrm{Xu} Z$, Mu J, Yang L, Gan H, Mu F, et al. Antibody response and viraemia during the course of severe acute respiratory syndrome (SARS)-associated coronavirus infection. Journal of Medical Microbiology,. 2003;53(5):435-8.

13. Young B, Woodford P, O'Dowd G. Wheater's Functional Histology E-Book: A Text and Colour Atlas. Elsevier Health Sciences; 2013. 467 p.

14. Blank F, Rothen-Rutishauser B, Gehr P. Dendritic Cells and Macrophages Form a Transepithelial Network against Foreign Particulate Antigens. Am J Respir Cell Mol Biol. 2007 Jun 1;36(6):669-77. 
15. Morgenroth K, Ebsen M. CHAPTER 8 - Anatomy. In: Papadakos PJ, Lachmann B, Visser-Isles L, editors. Mechanical Ventilation [Internet]. Philadelphia: W.B. Saunders; 2008 [cited 2020 May 19]. p. 69-85. Available from: http://www.sciencedirect.com/science/article/pii/B9780721601861500120

16. Ding $Y$, Wang $H$, Shen $H$, Li Z, Geng J, Han H, et al. The clinical pathology of severe acute respiratory syndrome (SARS): a report from China. The Journal of Pathology. 2003;200(3):282-9.

17. To KF, Lo AW. Exploring the pathogenesis of severe acute respiratory syndrome (SARS): the tissue distribution of the coronavirus (SARS-CoV) and its putative receptor, angiotensin-converting enzyme 2 (ACE2). The Journal of Pathology. 2004;203(3):7403.

18. Carsana L, Sonzogni A, Nasr A, Rossi R, Pellegrinelli A, Zerbi P, et al. Pulmonary postmortem findings in a large series of COVID-19 cases from Northern Italy. medRxiv. 2020 Apr 22;2020.04.19.20054262.

19. Varga Z, Flammer AJ, Steiger P, Haberecker M, Andermatt R, Zinkernagel AS, et al. Endothelial cell infection and endotheliitis in COVID-19. The Lancet. 2020 May 2;395(10234):1417-8.

20. Weiss SR, Navas-Martin S. Coronavirus Pathogenesis and the Emerging Pathogen Severe Acute Respiratory Syndrome Coronavirus. Microbiol Mol Biol Rev. 2005 Dec;69(4):635-64.

21. Hoffmann M, Kleine-Weber H, Schroeder S, Krüger N, Herrler T, Erichsen S, et al. SARSCoV-2 Cell Entry Depends on ACE2 and TMPRSS2 and Is Blocked by a Clinically Proven Protease Inhibitor. Cell. 2020 16;181(2):271-280.e8.

22. Li W, Moore MJ, Vasilieva N, Sui J, Wong SK, Berne MA, et al. Angiotensin-converting enzyme 2 is a functional receptor for the SARS coronavirus. Nature. 2003 Nov;426(6965):450-4.

23. Donoghue Mary, Hsieh Frank, Baronas Elizabeth, Godbout Kevin, Gosselin Michael, Stagliano Nancy, et al. A Novel Angiotensin-Converting Enzyme-Related Carboxypeptidase (ACE2) Converts Angiotensin I to Angiotensin 1-9. Circulation Research. 2000 Sep 1;87(5):e1-9.

24. Hamming I, Timens W, Bulthuis M, Lely A, Navis G, van Goor H. Tissue distribution of ACE2 protein, the functional receptor for SARS coronavirus. A first step in understanding SARS pathogenesis. J Pathol. 2004 Jun;203(2):631-7.

25. Harmer D, Gilbert M, Borman R, Clark KL. Quantitative mRNA expression profiling of ACE 2, a novel homologue of angiotensin converting enzyme. FEBS Letters. 2002;532(1-2):107-10.

26. Raj VS, Mou H, Smits SL, Dekkers DHW, Müller MA, Dijkman R, et al. Dipeptidyl peptidase 4 is a functional receptor for the emerging human coronavirus-EMC. Nature. 2013 Mar;495(7440):251-4.

27. The Human Protein Atlas. Tissue expression of DPP4 - Summary [Internet]. 2020 [cited 2020 May 19]. Available from: https://www.proteinatlas.org/ENSG00000197635DPP4/tissue 
28. de Wit E, van Doremalen N, Falzarano D, Munster VJ. SARS and MERS: recent insights into emerging coronaviruses. Nat Rev Microbiol. 2016;14(8):523-34.

29. Cong $Y$, Ren $X$. Coronavirus entry and release in polarized epithelial cells: a review. Reviews in Medical Virology. 2014;24(5):308-15.

30. Rossen JW, Bekker CP, Voorhout WF, Strous GJ, van der Ende A, Rottier PJ. Entry and release of transmissible gastroenteritis coronavirus are restricted to apical surfaces of polarized epithelial cells. J Virol. 1994 Dec;68(12):7966-73.

31. Rossen JWA, Voorhout WF, Horzinek MC, van der Ende A, Strous GJAM, Rottier PJM. MHV-A59 Enters Polarized Murine Epithelial Cells through the Apical Surface but Is Released Basolaterally. Virology. 1995 Jun 20;210(1):54-66.

32. Cong Y, Li X, Bai Y, Lv X, Herrler G, Enjuanes L, et al. Porcine aminopeptidase N mediated polarized infection by porcine epidemic diarrhea virus in target cells. Virology. 2015 Apr 1;478:1-8.

33. Lin X, O'Reilly KL, Storz J. Infection of polarized epithelial cells with enteric and respiratory tract bovine coronaviruses and release of virus progeny. Am J Vet Res. 1997 Oct;58(10):1120-4.

34. Schultze B, Zimmer G, Herrler G. Virus entry into a polarized epithelial cell line (MDCK): similarities and dissimilarities between influenza $C$ virus and bovine coronavirus. Journal of General Virology,. 1996;77(10):2507-14.

35. Pratelli A. Basic science track. Entry and release of canine coronavirus from polarized epithelial cells. New Microbiol. 2011 Jan;34(1):25-32.

36. Rossen JWA, Kouame J, Goedheer AJW, Vennema H, Rottier PJM. Feline and canine coronaviruses are released from the basolateral side of polarized epithelial LLC-PK1 cells expressing the recombinant feline aminopeptidase-N cDNA. Arch Virol. 2001 May 1;146(4):791-9.

37. Jia HP, Look DC, Shi L, Hickey M, Pewe L, Netland J, et al. ACE2 Receptor Expression and Severe Acute Respiratory Syndrome Coronavirus Infection Depend on Differentiation of Human Airway Epithelia. Journal of Virology. 2005 Dec 15;79(23):14614-21.

38. Pyrc K, Sims AC, Dijkman R, Jebbink M, Long C, Deming D, et al. Culturing the Unculturable: Human Coronavirus HKU1 Infects, Replicates, and Produces Progeny Virions in Human Ciliated Airway Epithelial Cell Cultures. Journal of Virology. 2010 Nov 1;84(21):11255-63.

39. Sims AC, Burkett SE, Yount B, Pickles RJ. SARS-CoV replication and pathogenesis in an in vitro model of the human conducting airway epithelium. Virus Res. 2008 Apr;133(1):33-44.

40. Tao X, Hill TE, Morimoto C, Peters CJ, Ksiazek TG, Tseng C-TK. Bilateral Entry and Release of Middle East Respiratory Syndrome Coronavirus Induces Profound Apoptosis of Human Bronchial Epithelial Cells. Journal of Virology. 2013 Sep 1;87(17):9953-8.

41. Tseng C-TK, Tseng J, Perrone L, Worthy M, Popov V, Peters CJ. Apical Entry and Release of Severe Acute Respiratory Syndrome-Associated Coronavirus in Polarized Calu-3 Lung Epithelial Cells. J Virol. 2005 Aug;79(15):9470-9. 
42. Wang G, Deering C, Macke M, Shao J, Burns R, Blau DM, et al. Human Coronavirus 229E Infects Polarized Airway Epithelia from the Apical Surface. Journal of Virology. 2000 Oct 1;74(19):9234-9.

43. Saltos FA, Saltos ADA. Entry of SARS-CoV2 through the Basal Surface of Alveolar Endothelial Cells \&ndash; A Proposed Mechanism Mediated by CD147 in COVID-19. 2020 May 23 [cited 2020 May 28]; Available from: https://www.preprints.org/manuscript/202005.0359/v1

44. Ibrahim IM, Abdelmalek DH, Elshahat ME, Elfiky AA. COVID-19 spike-host cell receptor GRP78 binding site prediction. Journal of Infection. 2020 May 1;80(5):554-62.

45. Li Y, Zhang Z, Yang L, Lian X, Xie Y, Li S, et al. The MERS-CoV Receptor DPP4 as a Candidate Binding Target of the SARS-CoV-2 Spike. iScience. 2020 Jun 26;23(6):101160.

46. Wang K, Chen W, Zhou Y-S, Lian J-Q, Zhang Z, Du P, et al. SARS-CoV-2 invades host cells via a novel route: CD147-spike protein. bioRxiv [Internet]. 2020 Mar 14 [cited 2020 May 19]; Available from: https://www.biorxiv.org/content/10.1101/2020.03.14.988345v1

47. Gordon DE, Jang GM, Bouhaddou M, Xu J, Obernier K, White KM, et al. A SARS-CoV2 protein interaction map reveals targets for drug repurposing. Nature. 2020 Apr 30;113.

48. George PM, Wells AU, Jenkins RG. Pulmonary fibrosis and COVID-19: the potential role for antifibrotic therapy. The Lancet Respiratory Medicine [Internet]. 2020 May 15 [cited 2020 May 28];0(0). Available from: https://www.thelancet.com/journals/lanres/article/PIIS2213-2600(20)30225-3/abstract 